Nathalie Killguß, Thomas Wittenberg*, Robin Rupp, Mathias Hofer, Daniela Franz, and Thomas Eixelberger

\title{
Interactive Image Segmentation for Cochlea Implant Planning based on DVT Data
}

https://doi.org/10.1515/cdbme-2019-0104

\begin{abstract}
Cochlea Implant (CI) planning is usually based on preoperative obtained CT or MRI data, visualising risk structures in the petrosal bone. In the past years, Digital Volume Tomography (DVT) with an increased spatial resolution and reduced radiation has become more important in the clinical routine for otology. In this work we propose an extension of our interactive "wizard"-guided approach for the interactive segmentation of the middle and inner ear structures for the use with DVT data. Different filter pipelines enable the user to interactive segment the acoustic canal, ossicles, tympanic cavity, facial nerve, chorda tympani, round window, cochlea and semicircular canals. The approach has been evaluated on six pre-operative acquired DVT datasets by an ENT expert. Results imply that the proposed method can handle DVT well and can potentially be used for interactive OR planning.
\end{abstract}

Keywords: Interactive Segmentation, Image processing, Digital Volume Tomography, Cochlea Implant planning.

\section{Introduction}

Cochlea Implant (CI) planning is usually based on preoperative obtained CT or MRI volume data. These data depict the risk structures in the petrosal bone, but the resolution is too low to recognise every small structure. Recently Digital Volume Tomography (DVT) has become more important in the clinical routine for otology. The benefits alongside the smaller voxel size are the shorter acquisition time and less radiation compared to normal CT scans. Thereby DVT is ideal for surgical planning of CI inventions. Nevertheless grey levels are not normalised to Hounsfield units and DVT depicts a higher level of noise. In order to support physicians with a preoperative visualisation and planning tool we propose an extension of our "Wizard"-guided approach for interactive segmentation

Nathalie Killguß, Thomas Wittenberg, Daniela Franz, Thomas Eixelberger, Fraunhofer Institute for Integrated Circuits IIS, Am Wolfsmantel 33, Erlangen, Germany, e-mail:

thomas.wittenberg@iis.fraunhofer.de

Robin Rupp, ENT Clinics University Erlangen, Erlangen, Germany

Mathias Hofer, University of Leipzig, Leipzig, Germany of middle and inner ear structures $[1,2]$ for the use with DVT data.

State of the Art: Not many approaches on ear structure segmentation in DVT data have been published until now. Some methods have been suggested in the dental area as e.g. jaw or teeth segmentation, as DVT is so far mostly used in dentistry. In [3] Pavaloiu et al apply edge detection in dental CBCT for teeth 3D-reconstruction, using a neural network. Loubele et al [5] present in a method to segment the jawbone and validate its quality in DVT data with the help of global thresholding and Gaussian mixture models. The authors of [4] describe a methodology for determining the length of the cochlea in CBCT data using the software tool OsiriX.

\section{Materials and methods}

The CI-Wizard [1, 2] provides an interactive segmentation of the individual ear structures in CT data. The three image planes (coronal, sagittal, coronal) and a 3D visualised view of the segmented ear structures are depicted to the user as visual feedback, cf. Fig. 1. For the segmentation of the middle and inner ear structures the user is lead by the CI-Wizard through eleven pages, thus step-by-step guiding the user through the semi-automatic segmentation process. Supported by the CIWizard, it is possible to extract the acoustic canal, the ossicles, tympanic cavity, facial nerve, chorda tympani, round window, cochlea and the semicircular canals. All structures are segmented using pre-defined filter pipelines, whose parameters can be changed interactively by the user on demand.

\subsection{Segmentation pipelines}

The original CT-based segmentation approach of the CIWizard is based on the Hounsfield Unit scale, but DVT data does not relate to this scale. Hence the filter pipelines for the segmentation had to be modified to support DVT image data. Additionally the cone beam effect affects the obtained image data and hence DVT data depict more noise than conventional CT data. In contrast to the CT-based approach, where spectral information from the Hounsfield units can be used, for the seg- 


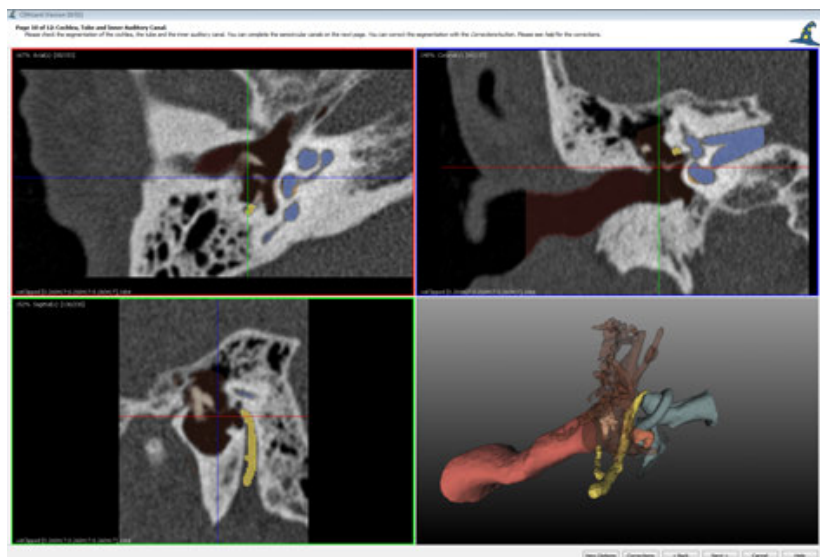

Fig. 1: User interface of the Cl-Wizard with segmented structures.

mentation of DVT data a lot of heuristics and thresholds must be applied.

Tab. 1: Modifications of the CT data based Cl-Wizard for using DVT data.

\begin{tabular}{lcc}
\hline Ear structure & $\begin{array}{c}\text { Parameter } \\
\text { adjustment }\end{array}$ & $\begin{array}{c}\text { Pipeline adaption } \\
\text { Acoustic canal }\end{array}$ \\
Ossicles & $\checkmark$ & $\checkmark$ \\
Tympanic cavity & $\checkmark$ & \\
Nerves & $\checkmark$ & \\
Round window & $\checkmark$ & \\
Cochlea & $\checkmark$ & $\checkmark$ \\
Semicircular canals & $\checkmark$ & $\checkmark$ \\
\hline
\end{tabular}

Tab. 1 shows the modification made to apply the original CI-Wizard in combination of DVT data. Checkmark symbolise that at least one filter in the related filter pipeline had been changed or adjusted, respectively at least one new filter has been inserted into the pipeline.

Acoustic canal: For the segmentation of the acoustic canal we implement the filter pipeline depicted in Fig. 2. First the air is segmented via threshold filter with a lower bound less than -1024 and an upper bound of -80 . As this filter not only detects the air in the acoustic canal but also some artefacts, the largest component is used. The edges are obtained using the Canny Edge detector and combined with a morphological closing filter. In the next step the segmentation mask is subtracted from the largest volume choose a volume with a range of $[500,2000] \mathrm{mm}^{3}$. In the last step the outer and the inner ear volumes are removed.

Ossicles: For the segmentation of the ossicles (stapes, incus, malleus) the user sets a seed point manually (Fig 3) and a region growing approach with a lower grey-level of 100 is

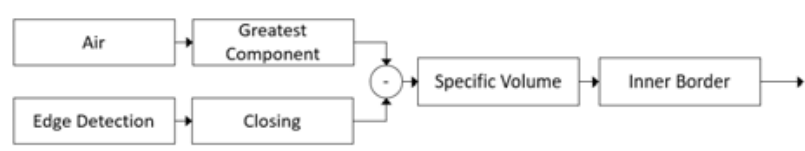

Fig. 2: Filter pipeline of the acoustic canal.

started. If the volume is smaller than $50 \mathrm{~mm}^{3}$, the segmented volume describes the ossicles, otherwise the threshold is decreased by 100 and the region growing starts again.

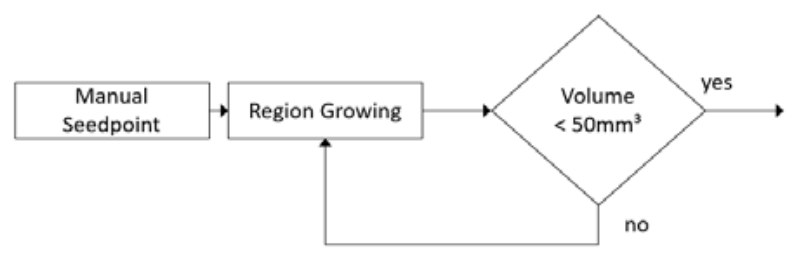

Fig. 3: Filter pipeline of the ossicles.

Tympanic cavity: Fig. 4 shows the filter pipeline for segmenting the tympanic cavity. We set a region of interest in dependency of the acoustic canal and perform a thresholding, with an upper limit of 40 . Next the largest connected component is chosen and subtracted from the acoustic canal. To this preprocessed data the volume of the ossicles is added. Finally, the volume in the range of $[430,1500] \mathrm{mm}^{3}$ is chosen.

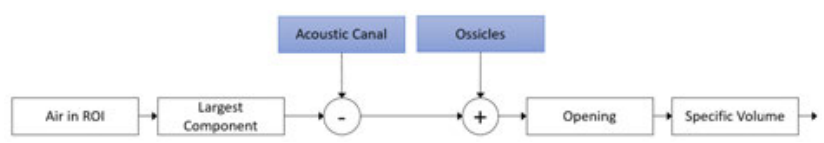

Fig. 4: Filter pipeline of the tympanic cavity.

Facial nerve and chorda tympani: The chorda tympani and the facial nerve are segmented interactively using a set of manually chosen seed points and a region growing processing. The thresholds are heuristically set to the range of [-245,345], while an additional boundary condition is the line diameter of $1.5 \mathrm{~mm}$.

Round window: For the segmentation of the round window we provide the user some possible candidates from which the user can select the best one (Fig. 5). To detect adequate candidates for the round window a vesselness filter [6] is applied in an automatically generated ROI using the segmented ossicles. This is united via a logic AND with the tympanic cavity and then connected with a threshold filter [1500, max]. Only volumes in the range of $[0.1,1.5] \mathrm{mm}^{3}$ are possible candidates for the round window.

Cochlea: In the filter pipeline (Fig 6) we first select a ROI according to the acoustic canal. With this ROI the voxels of 


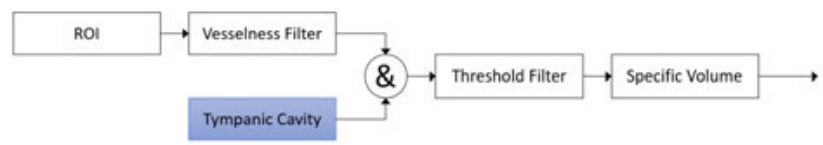

Fig. 5: Filter pipeline of the round window.

the tympanic cavity and the nerves are deleted. For this we need the DVT data, because we set the desired voxels to the lowest value of the DVT data. The successive threshold filter performs in the range of $[-400,400]$ and we choose the largest volume. We remove the border and select the largest connected volume. We add the round window and calculate the volume. If the volume is lower than $500 \mathrm{~mm}^{3}$ the cochlea is segmented, otherwise the threshold filter is increased iteratively by a value of 75 .

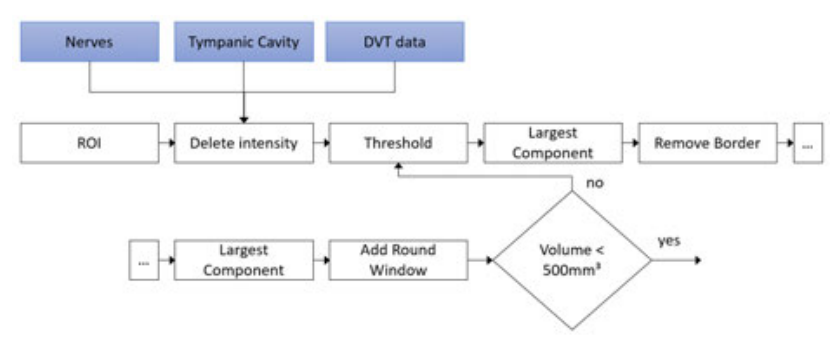

Fig. 6: Filter pipeline of the cochlea.

Semicircular canals: In the filter pipeline (Fig. 7) for the semicircular canals of the vestibular system the algorithm selects automatically a ROI depending on the previously segmented cochlea. A vesselness filter is used to obtain the semicircular canals. As this segmentation is not sufficient, a thresholding with a lower bound of 950 is used to obtain bright pixels. In parallel, a filter provides an internal seed point in one semicircular canal. The component in which the seed point lies is selected from the threshold mask. This mask includes air, round window and the facial nerve. Thus, the "air pixels" are subtracted and the segment is logically OR filtered with the cochlea volume. Now the largest connected component is chosen and the volumes of cochlea, round window and facial nerve are subtracted. Finally, the connected component is chosen.

\subsection{Evaluation}

The approach has been evaluated on six preoperative acquired DVT datasets by an ENT expert with four years of experience. Four patients depict normal ear anatomy, one shows a poorly ventilated tympanic cavity and one has a tympanoplasty. The

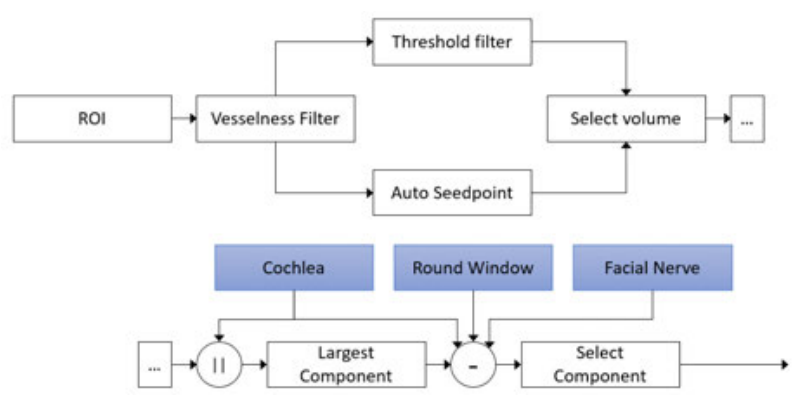

Fig. 7: Filter pipeline of the semicircular canals.

image data has been captured with a 3D Accuitoma DVT (J. Morita) and have a spacing of $0.125 \mathrm{~mm}$ in all three directions.

\section{Results}

The proposed segmentation runs mostly automatically. Only the volumes of the ossicles, nerves and round window need some interaction from the user. In the case of the ossicles, the user has to set only one seed point, while for the nerves, the user has to set more seed points. For the round window the user has to choose the right candidate out of a set of possibilities.

\subsection{Ear structures}

In all tested DVT datasets the eight different inner and middle ear structures could be segmented, while the complete process lasts 20 to 30 minutes depending of the anatomical knowledge of the user. Fig. 8 exemplary depicts all segmented structures provided by the CI-Wizard. In the upper left corner the axial view can be seen, the upper right provides the coronal slice while the lower left the sagittal slice is shown. The segmented structures are colorised in the slice views and in the lower right corner the user sees the rendered 3D models.

Fig. 9 shows the 3D model of one fully segmented dataset. The blueish models describe the cochlea and the semicircular canals. The light brown structure denote the ossicles and the brown transparent volume descries the tympanic cavity. The nerves are represented in yellow and the acoustic canal in reddish brown.

\subsection{Evaluation}

The ENT expert noticed that the nerves are not segmented automatically and a seed point has to be provided for the segmentation of the ossicles and a candidate for the round window. Also it was mentioned that the segmentation of the round 


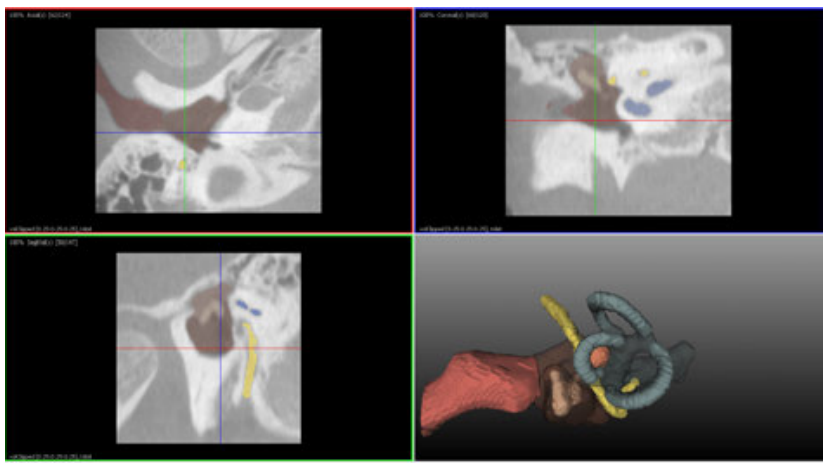

Fig. 8: User interface of the Cl-Wizard with a segmented DVT dataset.

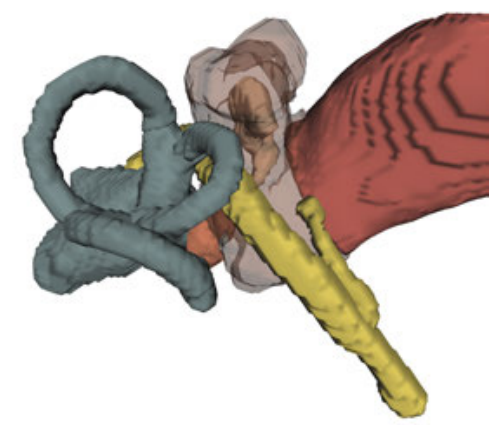

Fig. 9: 3D rendered models of the segmented structures.

window does not match the expected anatomical structure. The ossicles have not been segmented perfectly in some datasets. It was noted that the segmentation tool could also be a great tool for prospective ENT experts to learn the anatomical structures of the inner and middle ear and the location in the 3D space. Also the advantages of a segmentation before a surgery were stressed.

\section{Discussion}

We figured out that the described filter pipelines can (semi) automatically segment the risk structures of the middle and inner ear. According to the ENT expert some structures are not segmented perfectly yet. These inaccuracies in the area of the cochlea are induced by the volumetric quantisation of the data. The small segmentation inaccuracies of the ossicles are caused by blurred edges. This blur is induced by the higher noise compared to CT datasets. Hence, in future work a modeldriven approach for the segmentation of the ossicles could be used.

These shortcomings are not important for the CI intervention planning. The segmentation quality of the nerves depends on the knowledge of the user, similar wise for the round window and the ossicles. The round window does not represent the real anatomically structure, as the segmented area shows where a physician should insert the electrode into the cochlea. Thus the round window segmentation provides a guidance for the physician.

The proposed segmentation can also be used as an interactive anatomy learning software for prospective ENT experts. For this the tool provides a wizard-guided approach to segment the anatomical structures and explains the location of each structure in small text messages. Thus the user can read the text and check the location of the segmented structure in the next step.

Further work will evaluate the capability of this approach with additional and different data such as DVTs of child or special anatomies. The inaccuracies of the cochlea can further be removed by smoothing the extracted model and by the segmentation of the ossicles using a model-based approach. For the learning approach the round window pipeline has to be adjusted, that the anatomically structure is segmented and not the guidance for CI-planning.

\section{Author Statement}

This work was supported by the German Federal Ministry of Education and Research under the grant number 16SV7559.

\section{References}

[1] D Franz. Wizard-based image processing for the life sciences. Phd Thesis: FAU Erlangen-Nurember; 2016.

[2] D Franz, M Hofer, M Pfeifle, M Pirlich, M Stamminger, T Wittenberg. Wizard-based segmentation for cochlea implant planning. In Proc's Workshop Bildverarbeitung für die Medizin, pp.258-263; 2014.

[3] IB Pavaloiu, N Goga, A Vasilateanu, I Marin, A Ungar, I Patrascu, $C$ llie. Neural network based edge detection for CBCT Segmentation. EHealth and Bioengineering Conference (EHB), pp. 1-4; 2015.

[4] W Würfel, H Lanfermann, T Lenarz, O Majdan. Cochlear length determination using cone beam computed tomography in a clinical setting. Hearing research,316:65-72; 2014

[5] M Loubele, F Maes, F Schutyser, G Marchal, R Jacobs, $P$ Suetens. Assessment of bone segmentation quality of cone-beam ct versus multislice spiral CT: A pilotstudy. Oral Surgery, Oral Medicine, Oral Pathology, Oral Radiology and Endodontics, 102(2):225-234; 2006.

[6] AE Frangi, W J Niessen, K L. Vincken, M A. Viergever. Multiscale vessel enhancement filtering. Int. Conf. on medical image computing and computer-assisted intervention, pp. 130-137. Springer, Berlin, Heidelberg; 1998. 